\title{
Densidade amostral aplicada ao monitoramento georreferenciado de lagartas desfolhadoras na cultura da soja
}

\author{
Sample density applied to the georeferenced monitoring of defoliating caterpillars in soybean crop
}

\author{
Cinei Teresinha Riffel ${ }^{\mathrm{I}}$ Mauro Silveira Garcia ${ }^{\mathrm{II}}$ Antônio Luis Santi ${ }^{\mathrm{II}}$ Claudir José Basso $^{\mathrm{III}}$ \\ Lisandra Pinto Della Flora ${ }^{\mathrm{IV}}$ Maurício Roberto Cherubin ${ }^{\mathrm{v}}$ Mateus Tonini Eitelwein ${ }^{\mathrm{v}}$
}

\section{RESUMO}

\begin{abstract}
O conhecimento da distribuição espaço-temporal de insetos-praga na cultura da soja, por meio do uso de ferramentas da agricultura de precisão, tem sido apontado como uma importante estratégia no manejo integrado de pragas (MIP). Nesse sentido, o objetivo do trabalho foi avaliar a influência da densidade amostral no monitoramento de lagartas desfolhadoras na cultura da soja. O experimento foi conduzido em área experimental de 48, Oha, localizada no município de Júlio de Castilhos - RS, no ano agrícola 2008/ 2009. O monitoramento georreferenciado foi realizado seguindo três malhas amostrais regulares, 50x50m, 71x71m e $100 \times 100 m$ e também seguindo o método tradicional de amostragem. Durante todo o ciclo da cultura, e para cada malha amostral, foram realizadas cinco avaliações da infestação de lagartas, duas no estádio vegetativo e três no reprodutivo, com a utilização do pano-de-batida. Para analisar a distribuição espaço-temporal das lagartas na área, os dados foram submetidos à análise estatística descritiva e à geoestatística, utilizando semivariogramas e krigagem para elaboração dos mapas temáticos. Os resultados obtidos indicam que as grades amostrais avaliadas permitem caracterizar a distribuição espacial das lagartas e modelar a variabilidade espacial de lagartas na cultura da soja. A amostragem e monitoramento georreferenciado e posterior elaboração de mapas temáticos constituem-se numa alternativa potencial para agregarem-se às estratégias de MIP.
\end{abstract}

Palavras-chave: agricultura de precisão, malhas amostrais, Anticarsia gemmatalis, Pseudoplusia includens.

\section{ABSTRACT}

The knowledge of the spatial and temporal distribution of pest insects on soybean crop through the use of precision

\begin{abstract}
agriculture tools, have been appointed as an important strategy in the integrated pest management (IPM). In this sense, the objective of this research was to evaluate the influence of sample density in the monitoring of defoliating caterpillars in soybean crop. The experiment was conducted in the experimental area of 47.98ha, located in Júlio de Castilhos - RS city, in 2008/2009 season. The georeferenced monitoring was carried out following three regular grids, 50x50m, 71x71m and $100 \times 100 \mathrm{~m}$ and also following the traditional method of sampling. During the entire crop cycle, and for each grid, there were conducted five evaluations of caterpillar infestations, two on the vegetative stage and three in the reproductive, using a beat cloth. To analyze the spatial and temporal distribution of caterpillars in the area, the data were submitted to descriptive statistical analysis and geostatistics analysis, using semivariograms and kriging to the elaboration of thematic maps. The results obtained indicated that the evaluated sample grids allowed to characterize the spatial distribution of caterpillars and modeled the spatial variability of caterpillars in soybean crop. The sampling and the georeferenced monitoring and further development of georeferenced thematic maps constitute a potential alternative to aggregate to the IPM strategies.
\end{abstract}

Key words: precision agriculture, sample grids, Anticarsia gemmatalis, Pseudoplusia includens.

\section{INTRODUÇÃO}

A cultura da soja [Glycine $\max$ (L.) Merrill] caracteriza-se como uma das commodities mais importantes do agronegócio brasileiro, sendo cultivada na safra 2011/12 em praticamente 25 milhões de hectares, com produtividade média de $2.624 \mathrm{~kg} \mathrm{ha}^{-1}$

'Departamento de Agronomia, Sociedade Educacional Três de Maio (SETREM), Três de Maio, RS, Brasil.

IDepartamento de Fitossanidade, Faculdade de Agronomia Eliseu Maciel, Universidade Federal de Pelotas (UFPel), Pelotas, RS, Brasil.

"IIDepartamento de Ciências Agronômicas e Ambientais, Universidade Federal de Santa Maria (UFSM), 98400-000, Frederico Westphalen, RS, Brasil. E-mail: santi_pratica@yahoo.com.br. *Autor para correspondência.

${ }^{\mathrm{IV}}$ Colégio Agrícola de Frederico Westphalen (CAFW), UFSM, Frederico Westphalen, RS, Brasil.

'Programa de Pós-graduação em Agronomia, Agricultura e Ambiente, UFSM, Frederico Westphalen, RS, Brasil. 
(CONAB, 2012). No entanto, apesar da expressiva produção, a produtividade da soja ainda é limitada por diversos fatores abióticos e bióticos. Entre os últimos, destacam-se as pragas, que podem causar danos significativos se não manejadas eficientemente.

Dentre as pragas mais importantes na cultura, a lagarta-da-soja, Anticarsia gemmatalis Hübner, 1818 (Lepidoptera: Noctuidae), tem sido apontada como a principal desfolhadora, com danos que vão desde a desfolha parcial até a destruição completa da planta (PRAÇA et al., 2006). Além de $\boldsymbol{A}$. gemmatalis, outra importante lagarta desfolhadora que até pouco tempo era considerada uma praga secundária na cultura da soja e que atualmente exige controle é Pseudoplusia includens Walker, 1857 (Lepidoptera: Noctuidae), também conhecida como lagarta falsamedideira. A partir da safra 2003/2004, vários surtos de infestação dessa lagarta foram constatados em diversos estados brasileiros, tornando-a uma importante praga a ser controlada. O aumento da ocorrência de $\boldsymbol{P}$. includens deve-se, indiretamente, ao aumento considerável do número de aplicações de agrotóxicos na cultura da soja, que tem como consequência a diminuição do controle biológico natural desses insetos-praga por patógenos, parasitoides e predadores (BUENO et al., 2009; SOSA-GÓMEZ et al., 2010). Dentre os agrotóxicos, o uso de fungicida, especialmente para controle da ferrugem asiática, tem apresentado efeito negativo no desenvolvimento de fungos benéficos, como Nomuraea riley, causador da doença branca das lagartas (SOSA-GÓMEZ et al., 2003).

Nesse sentido, a compreensão de eventos da biologia de insetos-praga, relacionando-os a adaptações às diferentes condições ambientais e mudanças comportamentais que acontecem ao longo do ciclo de um inseto, deve ser reavaliada no intuito de estabelecer os padrões de densidades de insetos por área (MITCHELL \& FUXA, 1987). Dessa forma, o conhecimento da distribuição de uma população de insetos é indispensável para o desenvolvimento de protocolos de amostragem eficientes (TERRY et al., 1989).

Diante disso, no atual cenário de intensas transformações e inovações tecnológicas voltadas à agricultura, insere-se a agricultura de precisão, que permite, por meio do monitoramento georreferenciado e de técnicas geoestatísticas, o aprofundamento da dinâmica espaço-temporal dos insetos-praga nas diferentes áreas com culturas de interesse econômico (LIEBHOLD et al., 1993; WRIGHT et al., 2002; FARIAS et al., 2004; PARK \& TOLLEFSON, 2005; DINARDOMIRANDA et al., 2007; FARIAS et al., 2008; DAL PRÁ et al., 2011; PARK et al., 2011). A integração dessas inovações tecnológicas ao manejo integrado de pragas (MIP) pode contribuir na elaboração de protocolos para o monitoramento de pragas, bem como para o controle mais eficiente e localizado, reduzindo o custo de produção e a carga de agrotóxicos no ambiente.

Nesse sentido, o objetivo do trabalho foi avaliar a influência da densidade amostral aplicada ao monitoramento georreferenciado de lagartas desfolhadoras na cultura da soja.

\section{MATERIAL E MÉTODOS}

O estudo foi conduzido no ano agrícola 2008/2009, em área experimental localizada no município de Júlio de Castilhos, RS, Brasil, situada entre as coordenadas de latitude $29^{\circ} 27^{\prime} 33^{\prime \prime}$ S a 29 $29^{\prime} 38^{\prime \prime} \mathrm{S}$ e longitude $53^{\circ} 78^{\prime} 91^{\prime \prime}$ O a $53^{\circ} 80^{\prime} 14^{\prime \prime}$ 'O, totalizando 48, 0ha. O solo é classificado como Argissolo VermelhoAmarelo Alumínico típico, textura arenosa (EMBRAPA, 2006). O relevo da área é suave ondulado, com altitude aproximada de $513 \mathrm{~m}$. O clima da região é classificado, segundo MALUF (2000), como ST (subtropical), com temperatura média anual de $18,4^{\circ} \mathrm{C}$ e precipitação pluvial anual de $1.450 \mathrm{~mm}$.

A vetorização da área experimental foi obtida a partir da demarcação do perímetro, com o uso de aparelho de GPS de navegação portátil, marca Garmin ${ }^{\circledast}$, modelo Legend. Posteriormente, foram sobrepostas três malhas amostrais quadriculares regulares com as seguintes dimensões: a) 100x100m; b) 71x71m; c) 50x50m, totalizando 47; 96 e 181 pontos amostrais, respectivamente. As malhas amostrais foram geradas através do programa computacional CR - Campeiro 7 (GIOTTO \& ROBAINA, 2007). Com a finalidade de comparar os dados obtidos utilizando diferentes malhas amostrais georreferenciadas, em todas as avaliações, também foi realizada uma avaliação seguindo a metodologia tradicional com a utilização de pano-debatida (GALLO et al., 2002; PRAÇA et al., 2006).

O monitoramento das lagartas de $\boldsymbol{A}$. gemmatalis e de $\boldsymbol{P}$. includens foi realizado utilizando o método do pano-de-batida $(1,0 \times 0,5 \mathrm{~m})$, com três avaliações (repetições) em cada ponto, uma no ponto georreferenciado e as outras duas há aproximadamente $2 \mathrm{~m}$ à direita e esquerda desse ponto, paralelamente às linhas de cultivo. Ao longo do ciclo da cultura da soja, cinco avaliações foram realizadas para monitoramento das lagartas, nos seguintes estádios fenológicos da cultura: a) 1a avaliação, $\mathrm{V}_{4}$ (terceiro trifólio aberto); b) $2^{a}$ avaliação, $V_{6}$ (quinto trifólio aberto); c) 3a avaliação, $\mathrm{R}_{1}$ (início do florescimento); d) 4ª avaliação, $\mathrm{R}_{3}$ (início da formação de vagens); e) $5^{a}$ avaliação, $R_{5}$ (início de enchimento de grãos). 
Os dados foram submetidos à análise estatística descritiva, utilizando o programa computacional Statistical Analysis System - SAS 8.0 (SAS INSTITUTE, 1999). As análises da variabilidade espacial das distribuições das lagartas em função da dimensão da malha amostral foram realizadas através de semivariogramas experimentais (ISAAKS \& SRIVASTAVA, 1989). O ajuste de semivariogramas experimentais foi realizado por modelos teóricos utilizando o programa computacional GS+(ROBERTSON, 1998). Os modelos dos semivariogramas foram ajustados com base no melhor coeficiente de correlação obtido pela técnica chamada de validação cruzada.

Do ajuste de um modelo matemático aos dados, foram definidos os parâmetros do semivariograma: efeito pepita $(\mathrm{C} 0)$, patamar $(\mathrm{C})$, alcance (a), contribuição (C1). O grau de dependência espacial (GD) foi calculado por meio da equação $\mathrm{GD}=[\mathrm{CO} /(\mathrm{CO}+\mathrm{C} 1)]^{* 100}$, cuja classificação, segundo CAMBARDELLA et al. (1994), é forte, para GD $\leq 25 \%$; moderado, para GD entre 25 e $75 \%$, e fraco, para GD>75\%. O método de interpolação geoestatística utilizado para construção dos mapas temáticos foi a krigagem, efetuada por meio do programa CR-Campeiro7.

\section{RESULTADOS E DISCUSSÃO}

De maneira geral, observa-se que, em todas as avaliações, independente das malhas amostrais utilizadas, os dados apresentaram elevada dispersão relativa em relação à média, verificados pelos valores dos coeficientes de variação (CV\%), que variaram de 128,0\% ( $2^{a}$ avaliação) a 52,2\% (5 avaliação) na malha 50x $50 \mathrm{~m}$, de $130,0 \%$ ( $2^{\mathrm{a}}$ avaliação) a 50,3\% (5a avaliação), na malha 71x71m, e de 247,6\% (1 avaliação) a 49,2\% (5⿳亠口冋 avaliação), na malha 100x100m (Tabela 1). Dessa forma, quando o coeficiente de variação é muito alto (CV>30\%), os dados não revelam homogeneidade (PIMENTEL-GOMES \& GARCIA, 2002). A não homogeneidade dos dados justifica-se pelo número mínimo e máximo de lagartas observadas nas avaliações, que também variaram durante o ciclo da cultura, partindo de zero na 1 a avaliação até chegar a 20,3 lagartas por pano-de-batida na 5a avaliação. Esse aumento no número de lagartas desfolhadoras somente no final do ciclo da soja deve estar relacionado, dentre outros fatores, a não aplicação de inseticidas, visto que o nível de controle de 20 lagartas por pano-de-batida (SOSA-GÓMEZ et al., 2010) não foi atingido. No entanto, foi realizada uma aplicação de herbicida no estágio vegetativo e uma de fungicida no início do florescimento, que pode ter afetado negativamente a ocorrência de fungos entomopatogênicos, como $N$. riley.
Entretanto, verificou-se também que, à medida que aumentou o número de lagartas, especialmente na $5 \underline{a}$ avaliação, reduziram-se os valores de $\mathrm{CV} \%$, demonstrando maior homogeneidade na distribuição das lagartas na área. Ao avaliar a variação dos dados entre as três malhas amostrais utilizando o índice de variação (IV\%), que leva em consideração o número de repetições (PIMENTEL-GOMES \& GARCIA, 2002), verificou-se que, quanto menor a malha amostral (pontos amostrais mais próximos), mais acuradas são as avaliações realizadas ( $<\mathrm{IV} \%$ ), permitindo maiores níveis de acerto frente à distribuição das lagartas na área, a partir da amostragem realizada (Tabela 1).

Na tabela 2, são apresentados os resultados da análise geoestatística da distribuição de lagartas na cultura da soja, utilizando diferentes dimensões de malhas amostrais na área experimental de estudo. $\mathrm{Na} 1^{\underline{a}}$ avaliação, independente da malha amostral, não foi constatada a presença de lagartas na área. A partir da $2^{a}$ avaliação, a análise geoestatística permitiu caracterizar a variabilidade espacial e o padrão de distribuição de lagartas na área. O modelo pelo qual os semivariogramas foram melhores ajustados foi o esférico. Esse modelo é o mais aplicado nos estudos envolvendo parâmetros relacionados à entomologia, indicando que os insetos formam agregações no campo, ou seja, o ataque geralmente ocorre em reboleiras (WRIGHT et al., 2002; FARIAS et al., 2004; DINARDO-MIRANDA et al., 2007; FARIAS et al., 2008; DAL PRÁ et al., 2011).

A dependência espacial de todas as avaliações foi classificada, de acordo com CAMBARDELLA et al. (1994), como moderada, com valores do efeito pepita em relação ao patamar, ou seja, do grau de dependência (GD) variando de 51\% a 59\%. Os resultados da dependência espacial representam a influência dos pontos amostrados em relação aos pontos vizinhos. Quando existe dependência espacial entre os pontos amostrados, a distribuição espacial dos insetos é caracterizada como agregada e, nesse caso, a geoestatística é a ferramenta mais adequada para estudar as populações de insetos (LIEBHOLD et al., 1993). Resultados semelhantes aos obtidos no trabalho também foram encontrados em estudos com outras pragas agrícolas, tais como: Ostrinia nubilalis (Hübner) (Lepidoptera: Crambidae) (WRIGHT et al., 2002), Dilobopterus costalimai Young, Acrogonia sp. (Osborn) e Oncometopia facialis (Signoret) (Hemiptera: Cicadellidae) (FARIAS et al., 2004), Diabrotica virgifera virgifera LeConte (Coleoptera: Chrysomelidae) (PARK e TOLLEFSON, 2005), Mahanarva fimbriolata (Stål) (Hemiptera: Cercopidae) (DINARDO-MIRANDA et al., 2007), Spodoptera 
Tabela 1 - Análise estatística descritiva da distribuição de lagartas na cultura da soja, utilizando diferentes dimensões de malhas amostrais. Júlio de Castilhos, RS, Brasil, 2008/2009.

\begin{tabular}{|c|c|c|c|c|}
\hline \multirow{2}{*}{ Avaliações } & \multirow{2}{*}{ Parâmetros Estatísticos } & & lha Amos & \\
\hline & & $50 \times 50 \mathrm{~m}$ & $71 \times 71 \mathrm{~m}$ & $100 \times 100 \mathrm{~m}$ \\
\hline \multirow{8}{*}{$1^{\underline{a}}$} & $\mathrm{~N}^{0}$ de amostras & 181 & 96 & 47 \\
\hline & Mínimo & 0,00 & 0,00 & 0,00 \\
\hline & Máximo & 0,00 & 0,00 & 0,67 \\
\hline & Média & 0,00 & 0,00 & 0,04 \\
\hline & Desvio Padrão & 0,00 & 0,00 & 0,12 \\
\hline & Coeficiente de Variação & 0,00 & 0,00 & 247,61 \\
\hline & Índice de Variação & 0,00 & 0,00 & 36,12 \\
\hline & Erro & 0,00 & 0,00 & 79,36 \\
\hline \multirow{8}{*}{$2^{\mathrm{a}}$} & № de amostras & 181 & 96 & 47 \\
\hline & Mínimo & 0,00 & 0,00 & 0,00 \\
\hline & Máximo & 2,00 & 2,00 & 1,30 \\
\hline & Média & 0,31 & 0,38 & 0,26 \\
\hline & Desvio Padrão & 0,39 & 0,49 & 0,35 \\
\hline & Coeficiente de Variação & 127,9 & 129,94 & 130,66 \\
\hline & Índice de Variação & 9,51 & 13,26 & 19,06 \\
\hline & Erro & 18,68 & 26,13 & 37,76 \\
\hline \multirow{8}{*}{$3^{\underline{a}}$} & № de amostras & 181 & 96 & 47 \\
\hline & Mínimo & 0,00 & 0,00 & 0,00 \\
\hline & Máximo & 6,61 & 5,65 & 6,30 \\
\hline & Média & 1,21 & 1,11 & 1,14 \\
\hline & Desvio Padrão & 1,42 & 1,13 & 1,57 \\
\hline & Coeficiente de Variação & 117,64 & 101,84 & 137,57 \\
\hline & Índice de Variação & 8,74 & 10,39 & 20,07 \\
\hline & Erro & 17,19 & 20,48 & 39,66 \\
\hline \multirow{8}{*}{$4^{\underline{a}}$} & $\mathrm{~N}^{\mathrm{o}}$ de amostras & 181 & 96 & 47 \\
\hline & Mínimo & 0,00 & 0,00 & 0,00 \\
\hline & Máximo & 6,98 & 6,99 & 7,00 \\
\hline & Média & 1,51 & 1,53 & 1,46 \\
\hline & Desvio Padrão & 1,49 & 1,51 & 1,53 \\
\hline & Coeficiente de Variação & 98,30 & 98,76 & 104,56 \\
\hline & Índice de Variação & 7,31 & 10,08 & 15,25 \\
\hline & Erro & 14,36 & 19,86 & 30,26 \\
\hline \multirow{8}{*}{$5^{\underline{a}}$} & $\mathrm{~N}^{\mathrm{o}}$ de amostras & 181 & 96 & 47 \\
\hline & Mínimo & 1,11 & 1,34 & 1,33 \\
\hline & Máximo & 20,32 & 20,29 & 19,32 \\
\hline & Média & 8,40 & 8,47 & 8,82 \\
\hline & Desvio Padrão & 4,41 & 4,26 & 4,34 \\
\hline & Coeficiente de Variação & 52,20 & 50,33 & 49,25 \\
\hline & Índice de Variação & 3,88 & 5,14 & 7,18 \\
\hline & Erro & 7,67 & 10,12 & 14,23 \\
\hline
\end{tabular}

frugiperda (Smith) (Lepidoptera: Noctuidae) (FARIAS et al., 2008) e Diloboderus abderus Sturm (Coleoptera: Melolonthidae) (DAL PRÁ et al., 2011).
Quanto ao alcance, os valores variaram de 176 a $352 \mathrm{~m}$, na malha de $50 x 50 \mathrm{~m}$, de 124 a $372 \mathrm{~m}$, na malha de $71 \times 71 \mathrm{~m}$, e de 350 a $370 \mathrm{~m}$, na malha de 
Tabela 2 - Parâmetros geoestatísticos da distribuição de lagartas na cultura da soja, utilizando diferentes dimensões de malhas amostrais. Júlio de Castilhos, RS, Brasil, 2008/2009.

\begin{tabular}{|c|c|c|c|c|}
\hline \multirow{2}{*}{ Avaliações } & \multirow{2}{*}{ Parâmetros Geoestatísticos } & \multicolumn{3}{|c|}{--Malha Amostral-- } \\
\hline & & $50 \times 50 \mathrm{~m}$ & $71 \times 71 \mathrm{~m}$ & $100 \times 100 \mathrm{~m}$ \\
\hline \multirow{7}{*}{$1^{\underline{a}}$} & $\mathrm{~N}^{\mathrm{o}}$ de amostras & 181 & 96 & 47 \\
\hline & Modelo Ajustado & - & - & - \\
\hline & Efeito Pepita (Co) & - & - & - \\
\hline & Contribuição (C1) & - & - & - \\
\hline & Alcance $a(\mathrm{~m})$ & - & - & - \\
\hline & Patamar $(\mathrm{Co}+\mathrm{C} 1)$ & - & - & - \\
\hline & $\mathrm{GD}^{1}$ & - & - & - \\
\hline \multirow{7}{*}{$2^{\underline{\mathrm{a}}}$} & № de amostras & 181 & 96 & 47 \\
\hline & Modelo Ajustado & Esférico & Esférico & Esférico \\
\hline & Efeito Pepita (Co) & 0,078 & 0,133 & 0,070 \\
\hline & Contribuição (C1) & 0,076 & 0,110 & 0,049 \\
\hline & Alcance $a(\mathrm{~m})$ & 176 & 124 & 350 \\
\hline & Patamar $(\mathrm{Co}+\mathrm{C} 1)$ & 0,154 & 0,243 & 0,119 \\
\hline & GD & $51 \%$ & $55 \%$ & $59 \%$ \\
\hline \multirow{7}{*}{$3^{\mathrm{a}}$} & № de amostras & 181 & 96 & 47 \\
\hline & Modelo Ajustado & Esférico & Esférico & Esférico \\
\hline & Efeito Pepita (Co) & 0,914 & 0,671 & 1,439 \\
\hline & Contribuição (C1) & 1,114 & 0,598 & 1,029 \\
\hline & Alcance $a(\mathrm{~m})$ & 264 & 248 & 350 \\
\hline & Patamar $(\mathrm{Co}+\mathrm{C} 1)$ & 2,028 & 1,270 & 2,469 \\
\hline & GD & $45 \%$ & $53 \%$ & $58 \%$ \\
\hline \multirow{7}{*}{$4^{-\underline{a}}$} & $\mathrm{~N}^{\mathrm{o}}$ de amostras & 181 & 96 & 47 \\
\hline & Modelo Ajustado & Esférico & Esférico & Esférico \\
\hline & Efeito Pepita (Co) & 0,875 & 1,067 & 1,225 \\
\hline & Contribuição (C1) & 1,330 & 1,218 & 1,118 \\
\hline & Alcance $a(\mathrm{~m})$ & 352 & 372 & 350 \\
\hline & Patamar $(\mathrm{Co}+\mathrm{C} 1)$ & 2,205 & 2,286 & 2,343 \\
\hline & GD & $40 \%$ & $47 \%$ & $52 \%$ \\
\hline \multirow{7}{*}{$5^{\mathrm{a}}$} & $\mathrm{N}^{\mathrm{o}}$ de amostras & 181 & 96 & 47 \\
\hline & Modelo Ajustado & Esférico & Esférico & Esférico \\
\hline & Efeito Pepita (Co) & 8,621 & 8,526 & 9,646 \\
\hline & Contribuição (C1) & 10,823 & 9,660 & 9,236 \\
\hline & Alcance $a(\mathrm{~m})$ & 264 & 372 & 370 \\
\hline & Patamar $(\mathrm{Co}+\mathrm{C} 1)$ & 19,444 & 18,185 & 18,883 \\
\hline & GD & $44 \%$ & $47 \%$ & $51 \%$ \\
\hline
\end{tabular}

${ }^{1}$ GD: Grau de dependência espacial.

100x100m. O alcance é a distância limite de dependência espacial. Dessa forma, pontos de amostragem localizados a distâncias maiores que os valores de alcance apresentam distribuição aleatória, sendo independentes entre si (VIEIRA, 2000). Os dados de alcance podem auxiliar na escolha de uma malha amostral, em que a distância máxima entre os pontos forneça dados confiáveis de infestação por insetospraga nas áreas de cultivo (FARIAS et al., 2004). Dessa forma, verificou-se que as três malhas amostrais utilizadas no estudo foram eficientes para caracterizar a distribuição de lagartas $\boldsymbol{A}$. gemmatalis e $\boldsymbol{P}$. includens na cultura da soja.

Ciência Rural, v.42, n.12, dez, 2012. 
Observa-se, na figura 1 , que o aumento da densidade amostral, ou seja, ao se aproximarem os pontos amostrais, faz com que ocorra um maior detalhamento da distribuição espacial das lagartas na área e, consequentemente, gera mapas temáticos mais acurados. Esses dados corroboram a afirmação de COELHO et al. (2009), a qual aponta que, à medida que se aproximam os pontos amostrais, mais acurados são os valores dos locais não amostrados estimados por interpoladores geoestatísticos. Portanto, é esperado que pontos de amostragens localizados mais próximos sejam mais parecidos entre si do que os mais afastados, consequentemente, reduzindo a probabilidade de erros (VIEIRA, 2000). Por outro lado, à medida que aumenta a densidade amostral, aumenta o tempo necessário para executar o monitoramento e, consequentemente, os custos envolvidos na atividade, podendo tornar-se inviável à execução em áreas comerciais com grandes extensões. Dessa forma, cabe ao produtor a decisão de optar por utilizar uma malha maior ou menor, levando em consideração fatores como: tamanho da área, disponibilidade de mão-de-obra, estádio fenológico da cultura, níveis de infestação, dentre outros.
A espacialização dos valores obtidos na $5^{\text {a }}$ avaliação, momento de maior presença de lagartas na área, por meio de mapas temáticos (Figura 1), permitiu identificar as sub-regiões da área, com maior propensão a sofrer danos em função da presença de lagartas, independente da malha amostral utilizada. Embora, como já citado acima, o nível de acurácia na definição dessas sub-regiões dependerá diretamente da malha amostral utilizada, diferentemente do constatado na avaliação realizada pelo método tradicional (Figura 1d), que não permite a obtenção de tais informações e, consequentemente, a elaboração de estratégias de manejo mais pontuais na área.

Os dados encontrados reforçam as constatações de DAL PRÁ et al. (2011), já que conhecer a dinâmica espaço-temporal da praga pode proporcionar uma tomada de decisão melhor embasada, pela maior quantidade de informações obtidas e, como consequência, realizar o controle somente nas áreas com infestação. Além disso, este trabalho também endossa as afirmações já levantadas por ZAMBOLIN \& ZAMBOLIN (2008) de que a utilização de ferramentas de agricultura de precisão,

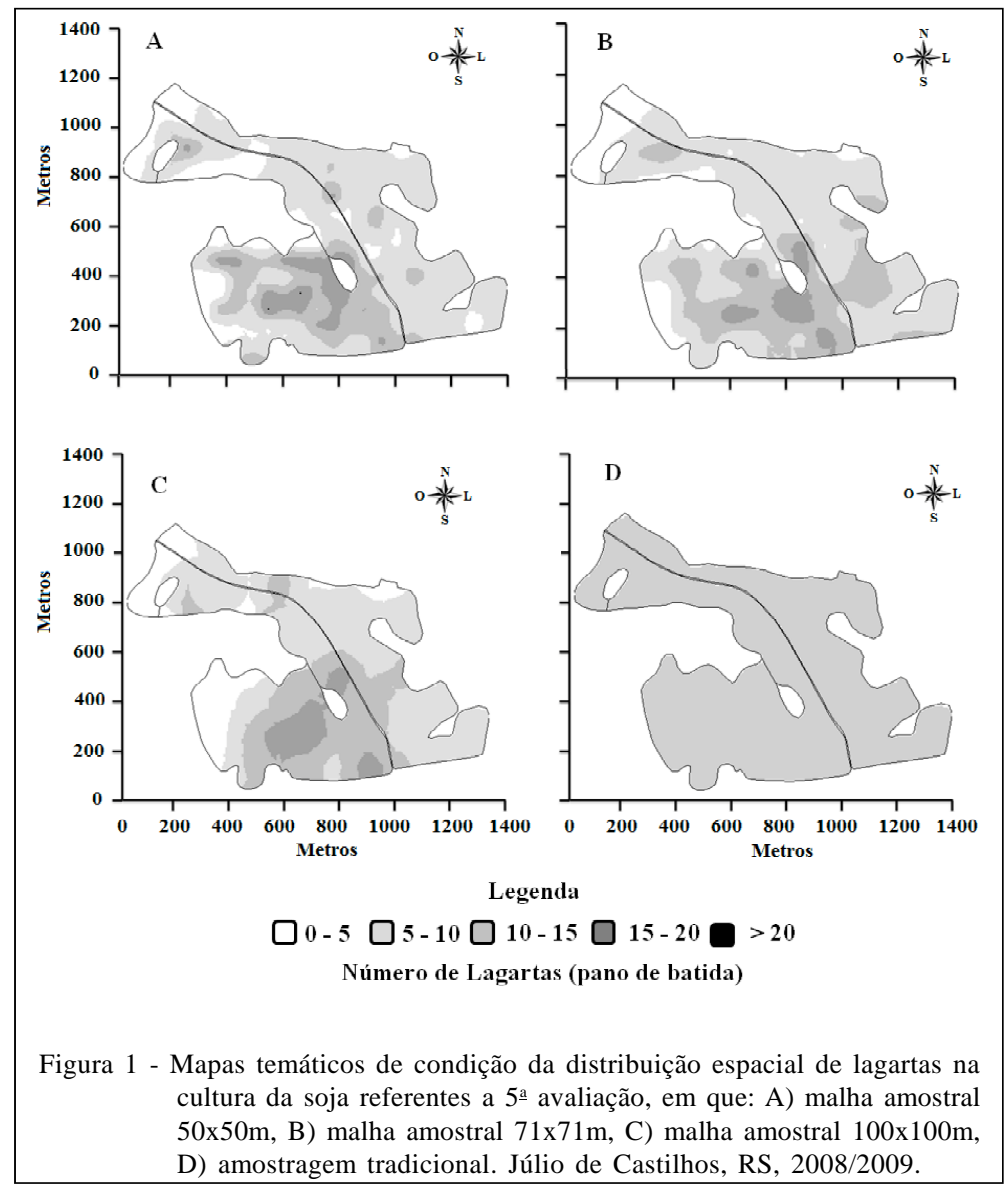

Ciência Rural, v.42, n.12, dez, 2012. 
como a aplicação em sítio específico, pode reduzir a quantidade de inseticidas utilizada. Portanto, a utilização de ferramentas de agricultura de precisão, tais como amostragem e monitoramento georreferenciado de lagartas na cultura da soja, permite traçar estratégias de controle, visando o uso racional de inseticida, gerando menores impactos ambientais.

\section{CONCLUSÃO}

As malhas amostrais avaliadas mostraramse apropriadas para caracterizar a distribuição espacial das lagartas na cultura da soja. No entanto, o aumento da densidade amostral aumenta a acurácia dos valores estimados e, consequentemente, dos mapas temáticos gerados.

As lagartas desfolhadoras A. gemmatalis e P. includens distribuíram-se de forma agregada na área, com moderada dependência espacial.

$\mathrm{O}$ monitoramento georreferenciado de lagartas na cultura da soja constitui uma alternativa potencial para futuros protocolos de controle de pragas, visando o manejo em sítios específicos e consequente redução nos impactos ambientais, gerados pela atividade agrícola.

\section{REFERÊNCIAS}

BUENO, R.C.O.F. et al. Desempenho de Tricogramatídeos como potenciais agentes de controle de Pseudoplusia includens Walker (Lepidoptera: Noctuidae). Neotropical Entomology, v.38, n.3, p.389-394, 2009. Disponível em: <http://www.scielo.br/ scielo.php?pid=S1519-566X2009000300015\&script=sci_arttext $>$. Acesso em: 15 jul. 2012. doi: 10.1590/S1519-566X2009000300015.

CAMBARDELLA, C.A. et al. Fieldscale variability of soil properties in central Iowa soils. Soil Science Society of America Journal, v.8, n.6, p.1501-1511, 1994. Disponível em: <http://ddr.nal.usda.gov./bitstream/10113/18168/1/ IND20518015.pdf >. Acesso em: 12 set. 2009.

COELHO, E.C. et al. Influência da densidade amostral e do tipo de interpolador na elaboração de mapas temáticos. Acta Scientiarum. Agronomy. v.31, n.1, p.165-174, 2009. Disponível em: <http://periodicos.uem.br/ojs/index.php/ ActaSciAgron/article/view/6645/6645>. Acesso em: 04 jun. 2011. doi: 10.4025/actasciagron.v31i1.6645.

CONAB - COMPANHIA NACIONAL DE ABASTECIMENTO. Levantamentos de safras. 2012. Disponível em: <http:// www.conab.gov.br/OlalaCMS/uploads/arquivos/ 12_04_11_15_04_18_boletim_abril_2012.pdf>. Acesso em: 05 maio, 2012.

DAL PRÁ, E. et al. Uso da geoestatística para caracterização da distribuição espacial de larvas de Diloboderus abderus. Ciência Rural, v.41, n.10, p.1689-1694, 2011. Disponível em: <http://www.scielo.br/ scielo.php?pid=S010384782011001000002\&script=sci_arttext $>$. Acesso em: 01 dez. 2011. doi: 10.1590/S0103-84782011001000002.
DINARDO-MIRANDA, L.L. et al. Uso da geoestatística na avaliação da distribuição espacial de Mahanarva fimbriolata em cana-de-açúcar. Bragantia, v.66, n.3, p.449-455, 2007. Disponível em: <http://www.scielo.br/pdf/brag/v66n3/ a11v66n3.pdf>. Acesso em: 08 nov. 2009. doi: 10.1590/ S0006-87052007000300011.

EMBRAPA - Empresa Brasileira de Pesquisa Agropecuária. Centro Nacional de Pesquisa de Solos. Sistema brasileiro de classificação de solos. 2.ed. Rio de Janeiro, 2006. 306p.

FARIAS, P.R.S. et al. Geostatistical characterization of the spatial distribution of Xylella fastidiosa sharpshooter vectors on citrus. Neotropical Entomology, v.33, n.1, p.13-20, 2004. Disponível em: <http://www.scielo.br/scielo.php?pid=S1519566X2004000100004\&script=sci_arttext $>$. Acesso em: 6 dez. 2009. doi: 10.1590/S1519-566X2004000100004.

FARIAS, P.R.S. et al. Spatial analysis of the distribution of Spodoptera frugiperda (Lepidoptera: Noctuidae) and losses in maize crop productivity using geostatistics. Neotropical Entomology, v.37, p.321-327, 2008. Disponível em: <http://www.scielo.br/ scielo.php?pid=S1519-566X2008000300012\&script=sci_arttext $>$. Acesso em: 12 dez. 2009. doi: 10.1590/S1519-566X2008000300012.

GALLO, D. et al. Entomologia agrícola. Piracicaba: FEALQ. 2002. 920p.

GIOTTO, E.; ROBAINA, A.D. A agricultura de precisão com o CR Campeiro 7. Manual do usuário. Santa Maria: UFSM/Centro de Ciências Rurais/Departamento de Engenharia Rural/Laboratório de Geomática, 2007. 319p.

ISAAKS, E.H.; SRIVASTAVA, R.M. An introduction to applied geostatistics. New York: Oxford University, 1989. 561p.

LIEBHOLD, A.M. et al. Geostatistics and geographic information system in applied insect ecology. Annual Review of Entomology, v.38, p.303-327, 1993. Disponível em: <http://www.annualreviews.org/ doi/pdf/10.1146/annurev.en.38.010193.001511>. Acesso em: 18 set. 2009. doi: 10.1146/annurev.en.38.010193.001511.

MALUF, J.R.T. Nova classificação climática do Estado do Rio Grande do Sul. Revista Brasileira de Agrometeorologia, v.8, p.141-150, 2000. Disponível em: <http://www.ufsm.br/ rba/p14181.html>. Acesso em: 07 set. 2009.

MITCHELL, F.L.; FUXA, J.R. Distribution, abundance, and sampling of fall armyworm (Lepidoptera: Noctuidae) in south central Louisiana corn fields. Environmental Entomology, v.16, p.453-458, 1987.

PARK, J.J. et al. Geostatistical analysis of the attractive distance of two different sizes of yellow sticky traps for greenhouse whitefly, Trialeurodes vaporariorum (Westwood) (Homoptera: Aleyrodidae), in cherry tomato greenhouses. Australian Journal of Entomology, v.50, p.144-151, 2011. Disponível em: <http://onlinelibrary.wiley.com/doi/10.1111/j.14406055.2010.00796.x/pdf >. Acesso em: 18 dez. 2011. doi: 10.1111/j.1440-6055.2010.00796.x.

PARK, Y.; TOLLEFSON, J.J. Spatial prediction of corn rootworm (Coleoptera: Chrysomelidae) adult emergence in Iowa cornfields. Journal of Economic Entomology, v.98, n.1, p.121-128, 2005. Disponível em: <http://www.bioone.org/doi/ 
abs/10.1603/0022-0493-98.1.121>. Acesso em: 16 dez. 2011. doi: 10.1603/0022-0493-98.1.121.

PIMENTEL-GOMES, F.; GARCIA, C.H. Estatística aplicada a experimentos agronômicos e florestais. Piracicaba: FEALQ, 2002. 309p.

PRAÇA, L.B. et al. Anticarsia gemmatalis Hübner, 1818 (Lepidoptera: Noctuidae). Biologia, amostragem e métodos de controle. Brasília: Embrapa Recursos Genéticos e Biotecnologia, 2006. 18p. (Documentos / Embrapa Recursos Genéticos e Biotecnologia, 0102-0110; 196).

ROBERTSON, G.P. GS+: geostatistics for the environmental sciences. Versão 5.03. Beta, Plainwell: Gamma Design Software, 1998. 152p.

SAS INSTITUTE - Statistical Analysis System. SAS/STAT User's Guide 8.0. North Caroline, NC, 1999. 3365p.

SOSA-GÓMEZ, D.R. et al. Soja: manejo integrado de pragas. Curitiba: SENAR-PR/EMBRAPA-Soja, 2010. 83p.

SOSA-GÓMEZ, D.R. et al. The impact of fungicides on Nomuraea rileyi (Farlow) samson epizootics and on populations of Anticarsia gemmatalis Hübner (Lepidoptera: Noctuidae), on soybean. Neotropical Entomology, v.32, n.2, p.287-291, 2003. Disponível em: <http://www.scielo.br/scielo.php?script=sci_arttext\&pid=S1519566X2003000200014>. Acesso em: 15 jul. 2012. doi: 10.1590/ S1519-566X2003000200014

TERRY, I. et al. Heliothis zea (Lepidoptera: Noctuidae) eggs in soybeans: within-field distribution and precision level sequential count plans. Environmental Entomology, v.18, p.908-916, 1989

WRIGHT, R.J. et al. Geostatistical analysis of the small-scale distribution of european corn borer (Lepidoptera: Crambidae) larvae and damage in whorl stage corn. Environmental Entomology, v.31, n.1, p.160-167, 2002. Disponível em: <http://www.cornpest.ca/index.cfm/linkservid/A9A8874465B8-8CF0-AB4B79959911AB59/showMeta/0/>. Acesso em: 08 nov. 2009. doi: 10.1603/0046-225X-31.1.160.

VIEIRA, S.R. Geoestatística em estudos de variabilidade espacial do solo. In: NOVAIS, R.F. et al. (Eds). Tópicos em Ciência do Solo. Viçosa: Sociedade Brasileira de Ciência do Solo, 2000. V.1, 352p. p.1-54.

ZAMBOLIN, L.; ZAMBOLIN, E.M. Manejo integrado de pragas. In: ZAMBOLIN, L. et al. (Eds). O que os engenheiros agrônomos devem saber para orientar o uso de produtos fitossanitários. $3 \mathrm{ed}$. Viçosa: UFV/DFP, 2008. Cap.VII, 464 p. p.259-359. 